\title{
Anisotropy effects and friction maps in the framework of the 2d PT model
}

\author{
O. Y. Fajardo ${ }^{\mathrm{a}, 1}$, E. Gnecco ${ }^{\mathrm{b}}$, J.J. Mazo ${ }^{\mathrm{a}, *}$ \\ ${ }^{a}$ Instituto de Ciencia de Materiales de Aragón and Departamento de Física de la Materia Condensada, CSIC-Universidad de Zaragoza, \\ E-50009 Zaragoza, Spain. \\ ${ }^{b}$ Instituto Madrileño de Estudios Avanzados, IMDEA Nanociencia, 28049 Madrid, Spain
}

\begin{abstract}
We present a series of numerical simulations on the friction-anisotropy behavior and stick-slip dynamics of a point mass in the framework of a 2d Prandtl-Tomlinson model. Results for three representative surface lattice are shown: square, hexagonal and honeycomb. Curves for scan angle dependence of static friction force, and kinetic one at $T=0 \mathrm{~K}$ and $T=300 \mathrm{~K}$ are shown. Friction force maps are computed at different directions.
\end{abstract}

Keywords: Friction, stick-slip, Tomlinson model, friction maps

\section{Introduction}

The one-dimensional Prandtl-Tomlinson model has been demonstrated to be a reference model to the theoretical study of friction at the atomic level $[1,2,3]$. It gives account for the universally observed stick-slip phenomena in friction and allows to qualitatively predict many of the characteristics of the observed friction vs velocity curves found in friction force microscopy experiments. Most studies using the PT model have been restricted to the $1 \mathrm{~d}$ domain. However, material surfaces are bidimensional, a fact that has unavoidable consequences. Perhaps, the most important feature is the dependence of the friction force with the direction of motion of the tip. Sometimes, it has been observed that the tip follows a straight line, which validates the use of a $1 \mathrm{~d}$ model in many cases. However, there exist other important situations where this is not the case.

Recent experiments have shown important variations in the atomic friction force for different sliding directions [4, $5,6]$. Two possible mechanisms for this effect have been proposed. On the one hand, commensurability and incommensurability effects in the sliding contatct can produce important differences in the friction force [7]. On the other hand, anisotropy effects arise due to the symmetry properties of the substrate [8].

Despite its importance, there are few systematic studies of the behavior of the friction force with the direction of sliding of the support. Because of experimental difficulties, such as sudden twisting of the tip termination [9] or wearing and contamination occurring after prolonged scanning, highly reproducible measurements of the frictional force with the scan angle are not currently available.

\footnotetext{
* Corresponding author

Email address: juanjo@unizar.es (J.J. Mazo)

${ }^{1}$ Present address: School of Chemistry, Tel Aviv University, Tel Aviv 69978, Israel.
}

Some experimental work at this respect have analyzed the behavior of the frictional force in quasi-crystalline surfaces $[10,11,9]$, and on molecular complexes [12]. Thus, theoretical studies of anisotropy in the friction force over a wide type of lattices are important sources of information to understand such effects.

\section{Model}

In the framework of the $2 \mathrm{~d}$ Prandtl-Tomlinson (PT) model, the motion of a sharp tip over the surface is described as the motion of a single particle of mass $m$ elastically coupled to a rigid support which moves over a $2 \mathrm{~d}$ surface in a given straight direction at certain velocity $\boldsymbol{v}_{s}(\boldsymbol{R})=\left(v_{x}, v_{y}\right)$. The scan direction of the support is given by the polar angle $\phi=\tan ^{-1}\left(v_{y} / v_{x}\right)$.

The dynamics of the system, including thermal effects, is described by a Langevin type of equation,

$$
m \frac{d^{2} \boldsymbol{r}}{d t^{2}}+m \gamma \frac{d \boldsymbol{r}}{d t}+\frac{\partial U(\boldsymbol{R}, \boldsymbol{r})}{\partial \boldsymbol{r}}=\xi(t)
$$

where $\boldsymbol{r}=(x, y)$ is the position vector of the tip and $\boldsymbol{R}(t)=\left(R_{x}, R_{y}\right)=\left(v_{x} t, v_{y} t\right)$ is the position of the support. The potential term $U(\boldsymbol{R}, \boldsymbol{r})=\frac{k}{2}[\boldsymbol{R}(t)-\boldsymbol{r}]^{2}+V(\boldsymbol{r})$ accounts for the effective elastic tip-support interaction and the tip-substrate potential. In our simulations we will use $v_{s}=25 \mathrm{~nm} / \mathrm{s}, m=2 \times 10^{-12} \mathrm{~kg}$ and a damping large enough to ensure overdamped dynamics of the system. It is common to measure the competition between the elastic and the substrate potential in terms of the parameter $\eta=4 \pi^{2} U_{0} / k a^{2}$, where $U_{0}$ is related to the amplitude of the substrate potential. We use $U_{0}=0.7 \mathrm{eV}$ and $\eta=7$ for all the cases.

Following the experimental procedure, in our simulations we will continuously move forth and back the support 


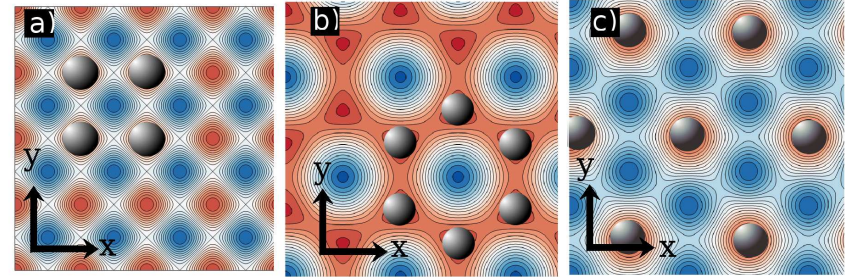

Figure 1: Underlying atomic structure and tip-substrate surface potential for a) the square; b) the hexagonal; and c) the honeycomb lattices. Note that the tip will try to move following the lattice minima positions and avoiding the surface atoms, that correspond to potential maxima.

tracing a series of scan lines and covering a square region of the surface. Different angles will be explored by rotating the substrate potential. It is important to mention that in some cases initial conditions dependence appear and results can be different from simulations made using other protocols.

We will restrict below to three types of surfaces shown in Fig. 1. The $\mathrm{NaCl}(001)$ surface can be modeled by a square symmetry potential

$$
V(x, y)=-U_{0}(\cos \tilde{x}+\cos \tilde{y}),
$$

where $\tilde{x}=2 \pi x / a$ and $\tilde{y}=2 \pi y / a$. The unit cell parameter $a=0.3988 \mathrm{~nm}$.

The second example we will study here is the case of highly oriented pyrolytic graphite (HOPG), which can be modeled with an hexagonal symmetry surface lattice [7], see Fig. 1(b). This potential can be written as

$$
V(x, y)=-U_{0}\left[2 \cos (\tilde{x}) \cos \left(\frac{\tilde{y}}{\sqrt{3}}\right)+\cos \left(\frac{2 \tilde{y}}{\sqrt{3}}\right)\right]
$$

with unit cell $a=0.246 \mathrm{~nm}$.

In addition we will study a surface with honeycomb symmetry [13], given by

$$
\begin{aligned}
& V(x, y)=0.80 U_{0}\left[\frac{3}{2}-\cos \left(\frac{\tilde{x}}{\sqrt{3}}\right) \cos \left(\frac{\tilde{y}}{3}\right)-\frac{1}{2} \cos \left(\frac{2 \tilde{y}}{3}\right)\right] \\
& \times\left[\frac{3}{2}+\cos \left(\frac{\tilde{x}}{\sqrt{3}}\right) \cos \left(\frac{\tilde{y}}{3}+\frac{\pi}{3}\right)+\frac{1}{2} \sin \left(\frac{2 \tilde{y}}{3}+\frac{\pi}{6}\right)\right] .
\end{aligned}
$$

In this case we also choose $a=0.246 \mathrm{~nm}$.

To determine the static friction force $\boldsymbol{F}_{\boldsymbol{s}}$, is enough to know the equilibrium positions of the point mass $m$ as a function of the support coordinates $[8,14]$. The stability region is obtained after resolving the equation given by $\nabla U(\boldsymbol{r})=0$ and the static friction force is reached when the tip position becomes unstable. Starting from this equation it is possible to derive relation for the static friction as a function of the support motion direction.

The kinetic friction of the system can be calculated at small or zero $T$ and for small velocities. In this case, the tip remains most of the time stuck to a potential minimum which becomes unstable at some point due to the motion of the support. Then the tip slides to another of the accessible minima of the system.

To determine the values of the kinetic friction force we have numerically solved the equation of the dynamics of the system and computed the value of the projection of the force $\boldsymbol{F}(\boldsymbol{r}, \boldsymbol{R})=k(\boldsymbol{R}(t)-\boldsymbol{r})$ along the direction of motion of the support

$$
F_{\text {proj }}=F_{x} \cos (\phi)+F_{y} \sin (\phi) .
$$

The kinetic friction is then computed as the mean value of this projection $F_{k}:=\left\langle F_{\text {proj }}\right\rangle$ (we average over around 4000 lattice constants).

\section{Results}

\subsection{Square lattice: the $\mathrm{NaCl}$ case}

Figure 2(a) shows the static (black squares) and the kinetic (blue circles for $0 \mathrm{~K}$ and red circles for $300 \mathrm{~K}$ simulations) for the $\mathrm{NaCl}$ case. In this case our numerical results can be compared to previous analytical ones. As shown in $[15,14]$ the static force in the $\eta \gg 1$ limit is given by

$$
F_{s}=\frac{2 \pi U_{0}}{a} \times \begin{cases}\frac{1}{\cos (\phi)} & \left(0^{\circ}<\phi<45^{\circ}\right) \\ \frac{1}{\sin (\phi)} & \left(45^{\circ}<\phi<90^{\circ}\right)\end{cases}
$$

Thus, it can be seen that the static force shows a minimum for $\phi=0^{\circ}$ and a maximum for $\phi= \pm 45^{\circ}$.

Regarding the kinetic friction force, its angle dependence can be written as [15]

$$
F_{k}=F_{k}\left(0^{\circ}\right)(\cos \phi+|\sin \phi|) \text {. }
$$

The kinetic friction force $F_{k}$ has a minimum for $\phi=0^{\circ}$ [this direction is marked as 1 in Fig. 2(a)] and a maximum at $\phi= \pm 45^{\circ}$ (direction 2 in figure). In Fig. 2(b) and (c) we can follow the tip motion in each case. For zero angle the tip follows a straight line from minimum to minimum over the saddle-point potential positions. This is different for other angles. In particular at $\pm 45^{\circ}$ as observed in the figure the tip advances following a zig-zag pattern avoiding potential maxima and exploring other regions of the potential landscape. A maximum value of the kinetic friction force is obtained for this particular direction. Comparing the maximum and minimum values of the static and kinetic friction, an anisotropy ratio $F_{\max } / F_{\min }=\sqrt{2}$ is found in both cases.

We have also made finite temperature simulations. If thermal effects are introduced in the system, a general decreasing of the friction force curve is observed. This reduction is due to the thermal activation of the tip over the overall potential barrier, which anticipates the slip events. Recent theoretical works [16], have found that the thermal behavior of the friction force in a square symmetry system shows a good agreement with $[T \ln (C T)]^{2 / 3}$ law, initially 

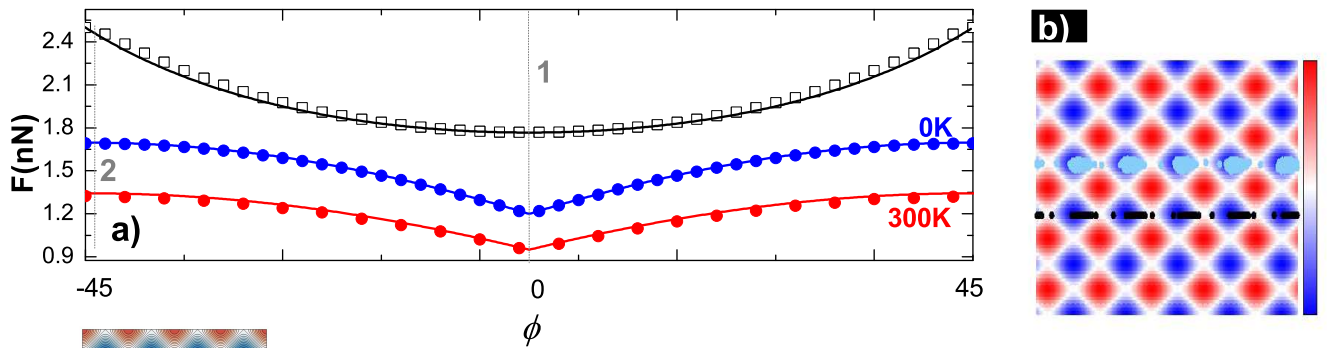

C)

1

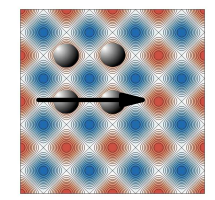

2
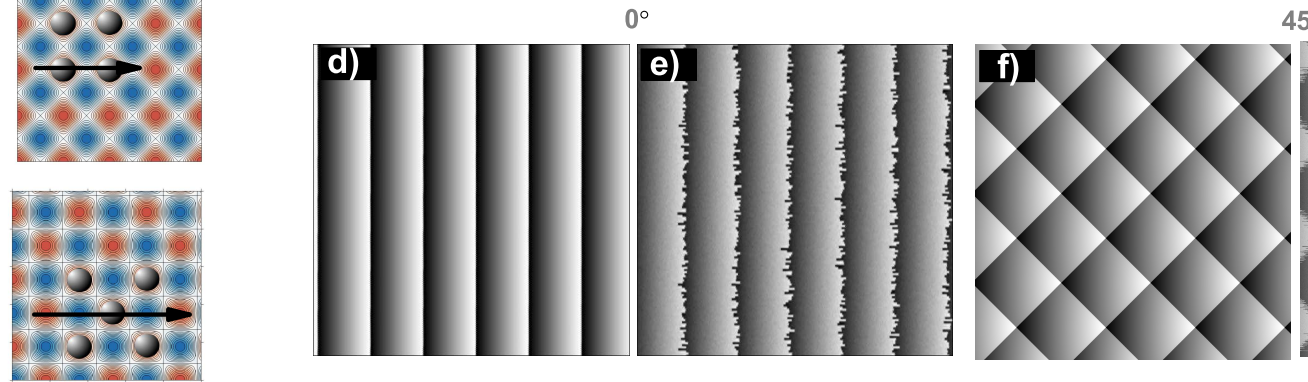

g)

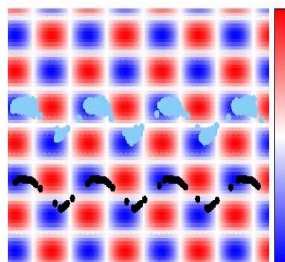

Figure 2: Square lattice results at 0 and $300 \mathrm{~K}$. a) Computed static (black squares) and kinetic (blue and red circles for 0 and $300 \mathrm{~K}$ respectively) friction force versus the scan angle. Lines correspond to theoretical predictions. Dashed vertical lines mark the $0^{\circ}$ (numbered as 1) and $45^{\circ}$ (line 2) directions, that correspond to maximum and minimum values of the force. b) and c) show traces of the tip trajectory at the two selected directions and for 0 and $300 \mathrm{~K}$. From d) to g) we show the computed friction maps at $0^{\circ}$ [ $\mathrm{T}=0 \mathrm{~K}$ in $\mathrm{d}$ ) and $\mathrm{T}=300 \mathrm{~K}$ in e)] and at $45^{\circ}[\mathrm{T}=0 \mathrm{~K}$ in $\mathrm{f}$ ) and $\mathrm{T}=300 \mathrm{~K}$ in $\left.\mathrm{g})\right]$.

ascribed to unidimensional systems. In $2 \mathrm{~d}$, thermal effects also introduce a dispersion in the tip trajectories which does not notably affect its basic pattern. Thus, the tip trajectories at $T=0 \mathrm{~K}$, are just disturbed by thermal noise at $T=300 \mathrm{~K}$, Fig. 2(b) and (c). In this case the global thermal effect is to decrease $F_{k}$ preserving the form of $F_{k}(\phi)$. This point is explicitly shown in Fig. 2(a) where we see that the angle dependence of the kinetic friction given in Eq. (7) is preserved. Thus an important anisotropy effect is also expected at $T=300 \mathrm{~K}$ in this lattice.

In addition we have computed the $2 \mathrm{~d}$ friction maps (without and with temperature) of this structure for the two chosen directions, following the procedure explained in Sec. 2, Figs. 2(d)-(g). The maps show the measured kinetic friction force as a function of the support position given by $\left(R_{x}, R_{y}\right)$. The map at $\phi=45^{\circ}$ of Fig. $2(\mathrm{f})$ clearly reproduces the structure of the underlying lattice. The presence of thermal effects just introduces an amount of noise in the resolution of the friction map, figure $2(\mathrm{~g})$. Regarding the $0^{\circ}$ direction, the situation is quite different. It can be seen that now the lattice structure is not totally shown, Figs. 2(d) and (e). The difference between both patterns can be understood from the differences observed in the tip motion for these configurations, figures $2(\mathrm{~b})$ and (c). For $45^{\circ}$, the tip shows a zig-zag motion. In the $0^{\circ}$ case the tip makes its "stick-slip" advance following a straight line, giving account of the friction map observed in Fig. 2.

\subsection{Hexagonal lattice; the HOPG case}

We present now results for an hexagonal lattice, see Fig. 1(b). Results are summarized in Fig. 3. In Fig. 3(a) we show the angle dependence of the static $F_{s}(\phi)$ (open square) and kinetic $F_{k}(\phi)$ (blue circles for $0 \mathrm{~K}$ and red ones for $300 \mathrm{~K}$ ) friction force. The first thing that we note is that the static force shows a weak dependence on the scan direction, a behavior quite different from the one found for the square lattice. According to our numerical results $F_{s, \max } / F_{s, \min }=1.015$. Similar small variations in the angle dependence of $F_{s}$ has been recently reported for smaller $\eta$ values, $(\eta \approx 2)$ [8].

The kinetic friction force $F_{k}(\phi)$ shows two singularities at $\phi=0^{\circ}$ and $\phi=30^{\circ}(\eta=7)$. First one corresponds to a straight motion of the tip, Fig. 3(b). The second singularity is associated with a zig-zag motion where tip alternates advances at $0^{\circ}$ and $60^{\circ}$, Fig. 3(c). Excluding the singularities, $F_{k}$ increases from $\phi=0^{\circ}$ to $\phi=30^{\circ}$ with $F_{k, \max } / F_{k, \min } \simeq 1.09$. This value is larger than the one found for the static case but smaller than the one obtained for the square lattice. Verhoeven et al. [7] reported larger variation in the kinetic force (up to $20-30 \%$ ) in numerical calculations for a sheet of material with hexagonal symmetry and 93-atoms in the tip apex.

The addition of thermal effects reduce the value of the kinetic friction force. At $300 \mathrm{~K}$ we still observe singularities at the $0^{\circ}$ direction (and equivalent ones), but strongly reduced. The one found at $\phi=30^{\circ}$ has nearly disappeared. In the same figure, panels (d) to (g) show the friction force maps at the different angles and temperatures.

We have to mention that the two super-cells pattern reported by Sasaki et al. [17] for hexagonal lattice friction maps is not observed in our simulations. The reason is 


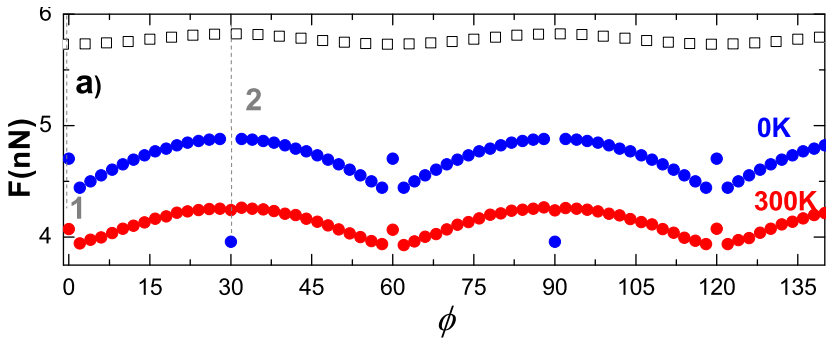

1
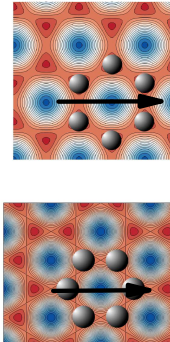

$0^{\circ}$

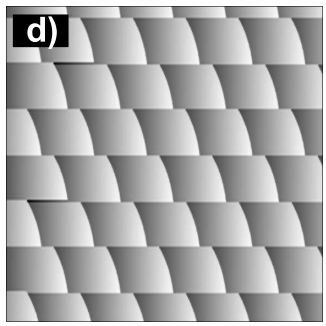

\section{b)}

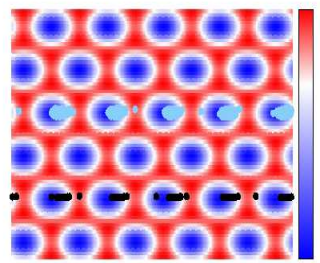

c)

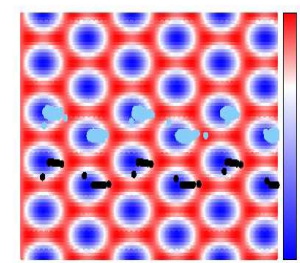

$30^{\circ}$
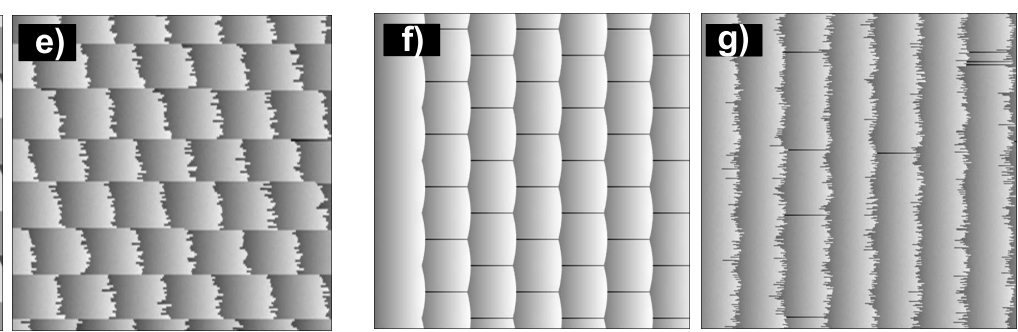

Figure 3: Angle dependence of the friction for an hexagonal lattice at $T=0$ and $T=300 \mathrm{~K}$. (a) Static, $F_{s}$ (squares), and kinetic, $F_{k}$ (circles) friction force. Singularities in the kinetic case are observed for $\phi=0^{\circ}$ (and equivalent values), $30^{\circ}$ (and equivalent values). The structure has a rotational symmetry of $60^{\circ}$. Dashed vertical lines show two specific directions labeled as $1\left(0^{\circ}\right)$ and $2\left(30^{\circ}\right)$. (b) and (c) show the trajectories for the selected directions at 0 and $300 \mathrm{~K}$. (d)-(g) are friction maps at the selected angles and temperatures. $\eta=7.0$ in all simulations.

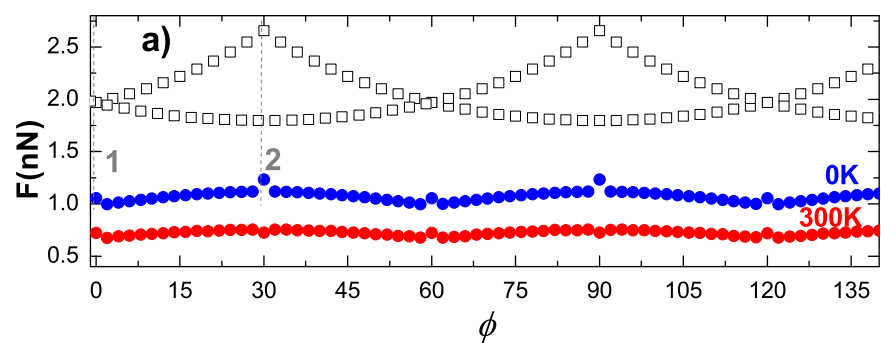

$0^{\circ}$ b)

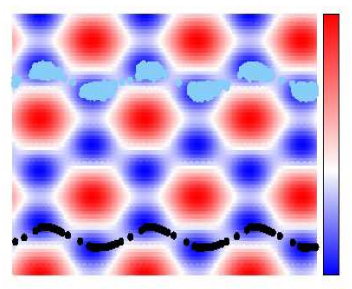

C)

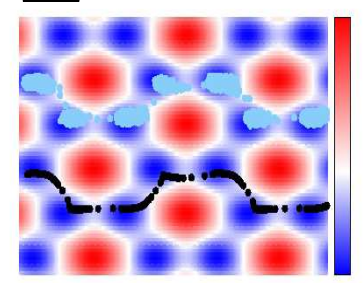

$30^{\circ}$
1

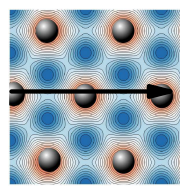

2

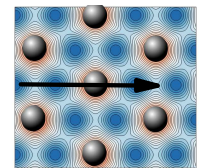

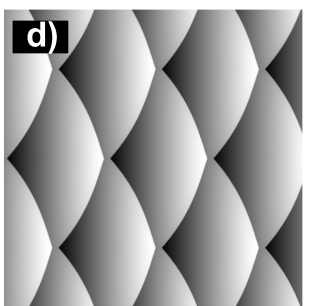

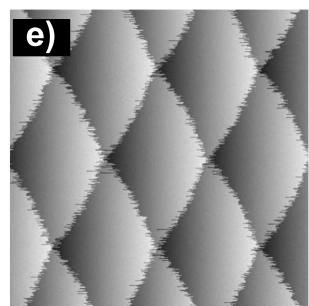

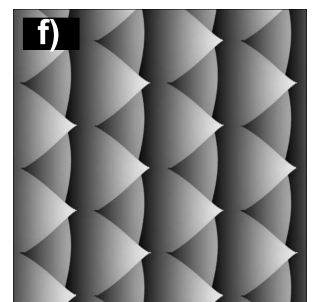

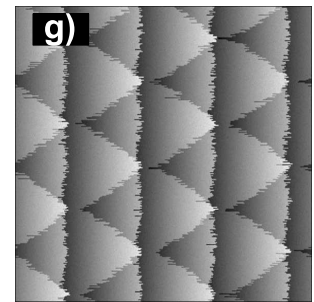

Figure 4: Friction force of the honeycomb structure. (a) Angle dependence of static (squares) and kinetic (circles) friction force. Dashed vertical lines label two different directions for which singularities in the kinetic friction force are found. (b) and (c) show trajectories of the tip for $\phi=0^{\circ}$ and $30^{\circ}$ at $0 \mathrm{~K}$ and $300 \mathrm{~K}$ respectively. (d)-(k) Friction force maps for four directions at 0 and $300 \mathrm{~K}$. In all the cases $\eta=7$.

the use of a different procedure to trace the maps. We have checked that this structure appears when friction map is traced scanning only the forth direction of the friction loops.

\subsection{Honeycomb lattice:}

We will show now results for a more complex structure, a honeycomb type which can be obtained from a hexagonal lattice with two atoms per unit cell, Fig 1(d). Figure 4(a) shows the dependence of the static force $F_{s}$ (squares) and the kinetic one $F_{k}$ (circles) as a function of the scan angle $\phi$. Dashed vertical lines show two different values of the angle, corresponding to different symmetry directions of the structure. Static friction force shows a $120^{\circ}$ period. Due to the existence of two atoms in the unit cells the value of the friction force depends on the 
chosen initial potential minimum and two, $60^{\circ}$ displaced one from each other, identical curves appear. Anisotropy effects are strong here since we find $F_{s, \max } / F_{s, \min } \simeq 1.48$, a large value. Regarding the kinetic force $F_{k}$, apart from the observed singularities, the observed anisotropy ratio $F_{k, \max } / F_{k, \min } \simeq 1$.11. Thermal effects reduce friction and reduce the singularities. Figures $4(\mathrm{~b})$ and (c) show two trajectories $\left(0^{\circ}, 30^{\circ}\right)$ at 0 and $300 \mathrm{~K}$.

To finish our study, Figs. 4(d)-(g) show the friction force maps for the two given angles: $\phi=0^{\circ}[(\mathrm{d})(0 \mathrm{~K})$ and (e) $(300 \mathrm{~K})]$ and $\phi=30^{\circ}$, figures $4(\mathrm{f})$ and (g). In a similar way that in the previous cases (square and hexagonal structures), these differences can be understood analyzing the point position distributions which result from the tip dynamics. Figure 4(b) and (c) show such distributions for $\phi=0^{\circ}$ and $\phi=30^{\circ}$. Evident differences in the point position are found. For $\phi=30^{\circ}$ there exists two different patterns meanwhile for $\phi=0^{\circ}$ a single one is observed.

\section{Discussion and conclusions}

In conclusion, we have extended recent works where the PT model was used to study the angle dependence of the static and kinetic friction force of different surfaces. We have also include thermal effects in our simulation work.

We see that the PT model is able to reproduce a series of interesting effects: from the strong anisotropy of the square lattice (when comparing to the hexagonal one) to the existence of a variable number of peaks in the friction curve depending on the scan angle. Such predictions can be experimentally studied only by detailed AFM measurements. Ultra-high vacuum conditions are required to measure static and kinetic friction force variations at different angles.

An important point to consider is the occurrence of tip-apex changes when the scan direction is varied. Unfortunately such problems are hard to avoid in an experiment. An interesting possibility is to rotate the sample via piezoelements without breaking the vacuum conditions [18]. Maintaining the cantilever and surface orientation and changing the sliding direction introduce problems, as perturbations coming from the buckling of the cantilever. This problem has been revisited in a recent work by Campione et al. [19], where apparent variations in the elevation produced by buckling were related to the friction component along the cantilever axis and used to determine the so-called hodograph friction of the surface.

\section{Acknowledgments}

The authors acknowledge financial support from Spanish MINECO through Project No. FIS2011-25167, cofinanced by FEDER funds and Project No. MAT201234487. O. Y. F. acknowledges financial support from FPU grant by Ministerio de Ciencia e Inovación of Spain.

\section{References}

[1] E. Gnecco and E. Meyer (Eds.), Fundamentals of friction and wear on the nanoscale, Springer (2007).

[2] Y. Dong, A. Vadakkepatt, and A. Martini, Tribol. Lett. 44, 367 (2011)

[3] A. Vanossi, N. Manini, M. Urbakh, S. Zapperi, and E. Tosatti, Rev. Mod. Phys. 85, 529 (2013).

[4] M. Dienwiebel et al., Phys. Rev. Lett. 92, 126101 (2004)

[5] S. J.Choi et al. Science 333, 607 (2011).

[6] C. M. Pina, R. Miranda, and E. Gnecco, Phys. Rev. B 85, 073402 (2012).

[7] G. Verhoeven, M. Dienwiebel, and J. Frenken, Phys. Rev. B 70, 165418 (2004).

[8] E. Gnecco, Europhys. Lett. 91, 66008 (2010).

[9] A. F. filippov, A. Vanossi, and M. Urbakh, Phys. Rev. Lett. 104074302 (2010).

[10] J. Y. Park et al., Phys. Rev. B 72220201 (2005).

[11] J. Y. Park et al., Phys. Rev. B 74024203 (2006).

[12] G. Fessler et al. Appl. Phys. Lett. 98, 083119 (2011).

[13] O. M. Braun, and R. Ferrando, Phys. Rev. E 65, 061107 (2002)

[14] E. Gnecco, O. Y. Fajardo, C. M. Pina, and J. J. Mazo, Tribol. Lett. 48, 33 (2012).

[15] P. Steiner et al. Phys. Rev. B 82, 205417 (2010)

[16] R. Roth et al., Phys. Rev. B 79045414 (2009).

[17] N. Sasaki, K. Kobayashi, and M. Tsukada, Phys. Rev. B 54, 2138 (1996).

[18] G. Fessler, private communication.

[19] M. Campione, S. Trabattoni, and M. Moret. Trib. Lett. 45, 219224 (2012). 